\title{
Experimental based Performance Analysis of Proactive OLSR, Reactive Tora and Hybrid GRP Routing Protocols in MANET
}

\author{
Muhammad Asif Mehmood \\ Khan \\ Student MS (CS), CS Department \\ University of Agriculture, \\ Faisalabad
}

\author{
Ahmed Mateen Buttar \\ Lecturer, CS Department \\ University of Agriculture, \\ Faisalabad
}

\author{
Muhammad Ashraf \\ Computer Teacher \\ Education Department
}

\begin{abstract}
Mobile Ad hoc Network (MANET) contains mobile nodes which exchange information dynamically over wireless links. Routing Protocols are the most vital element of MANET, which are needed to handle dynamic communication and also find route. Performance of routing protocols is an important issue. In MANET, due to mobility of nodes, the wireless links are highly error prone and can go down frequently, interference and less infrastructure. In this research, performance of Optimized Link State Routing (OLSR), Temporary Ordered Routing Algorithm (TORA) and Geographic Routing Protocol (GRP) routing protocols are evaluated in mobile Ad hoc network by using simulator against various quantitative metrics like Network Load, Throughput, Retransmission Attempts and Media Access Delay by varying physical characteristics, number of nodes, nodes speed and pause time. Various simulation scenarios are investigated by varying matrices. A comparative performance analysis of aforesaid protocols is carried out in this research. In the last, conclusion is presented which demonstrates that performance of routing protocols differs by varying matrices.
\end{abstract}

\section{Keywords}

MANET, OLSR, TORA, GRP, FTP.

\section{INTRODUCTION}

The most well-known wireless networks categorised in two types first one is Infrastructure less and second is Infrastructure. The first one type of wireless network can be in motion at the same time as correspond while the base stations are remain fixed in this wireless network and the range of nodes go out from a base station, then nodes find the range of a further base station automatically [1].

MANET is a type of automatically configuring network of linked nodes and MANET routers connected with wireless links, this way of combination in which MANET routers establishes a random topology network. MANETs are habitually built a short term set up in urgent situation. This type of network work in the nonappearance of every set infrastructure that makes it simply to install at the similar time, on the other hand it turn into hard to utilize the available routing method to network services due to the nonappearance of any fixed infrastructure and it has a many challenges in make sure the safety of the communication, it is not simply completed as several difficulty of network security clash by the requirements of mobile networks, essentially owing to the environment to the mobile hosts e.g. utilization of low power, small processing load [2]. Proactive protocols are those which always maintain a route to every possible destination while reactive protocols are those that discover and maintain a route to a destination only when one is required [3]. While the third type of MANAT routing protocols is hybrid which is mixture of proactive and reactive routing protocols [4].

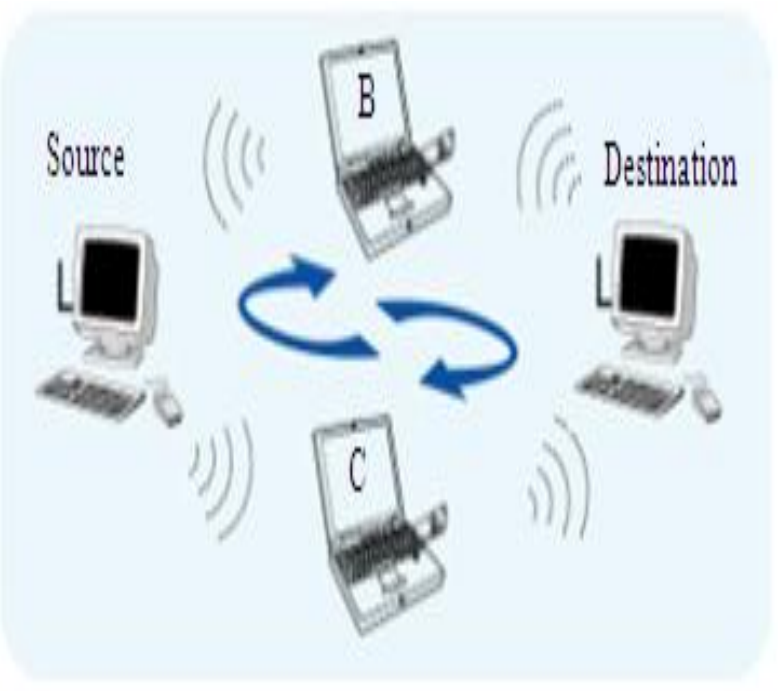

Fig 1: MANET

\subsection{Classification of routing protocols in ad hoc networks.}

Ad hoc protocols are divided in the following categories

- $\quad$ Flat Routing Protocols

$$
\begin{aligned}
& \text { ○ Reactive Routing Protocols } \\
& \text { ○ Proactive Routing Protocols }
\end{aligned}
$$

- Hybrid Routing Protocols

- Hierarchical Routing Protocols

- Geographical Routing Protocols 


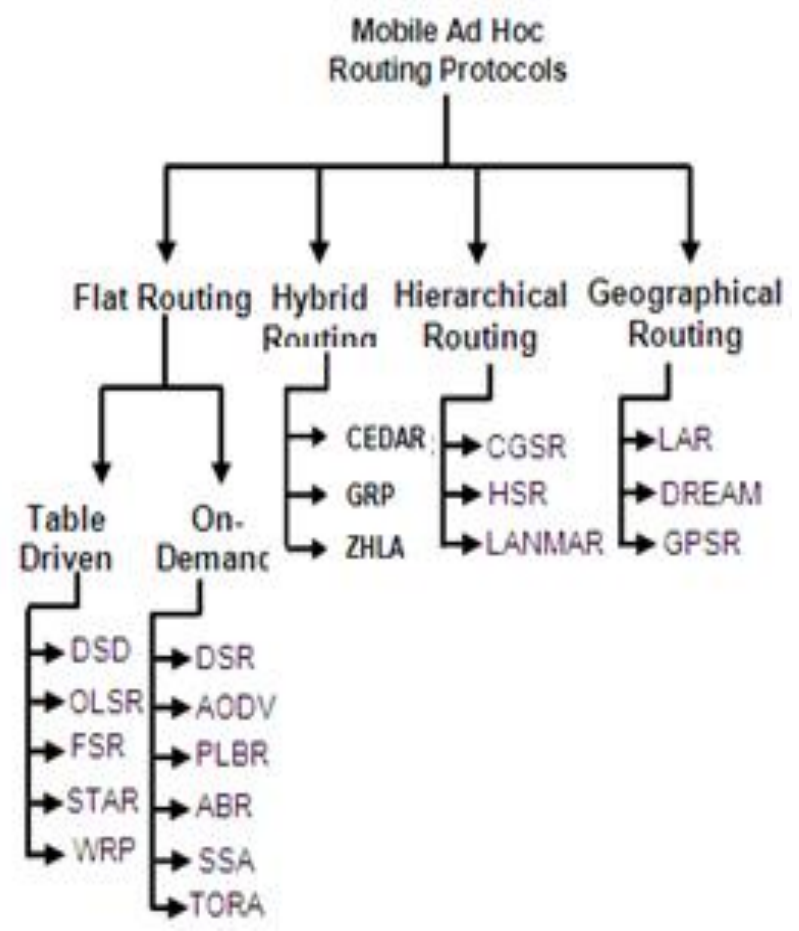

Fig 2: Classification of Routing Protocols

\subsubsection{Optimized Link State Routing}

Optimized Link State Protocol (OLSR) is a recognized as proactive routing protocol, therefore the direction are for all time instantaneously existing. OLSR is an optimization edition of a pure link state protocol. The topological be different by causes the flooding of the nodes information to each and every accessible nodes in the set-up. To decrease the promising overhead in the network protocol makes use of Multipoint Relays. The scheme of MPR is to decrease flooding of broadcasts by reducing the similar broadcast in a few regions in the network. In additional it reduce to provide the shortest way [5]

\subsubsection{Temporally Ordered Routing Algorithm}

TORA work on the bases on link reversal, it is adaptive and scalable routing algorithm. TORA discover several ways from sender to receiver in a extremely dynamic mobile networking atmosphere. A significant thought of TORA is to facilitate control messages are localized to a small set of hosts close to a topological variation. Nodes keep up routing information regarding their instantaneous one hop neighbours. TORA has work with three basic functions e.g first one route creation, $2^{\text {nd }}$ one route safeguarding and last is route deletion. The each metric of this protocol has include five part that are unique node ID, logical time of a link failure, the unique ID of a node that defined the new reference level, a reflection indicator bit, and a propagation ordering parameter [5].

\subsubsection{Geographic Routing Protocol}

GRP is a position based protocol classified as hybrid routing protocol. In GRP the Global Positioning System (GPS) is used to mark the location of node and the quadrants optimize flooding. When a node moves and crosses neighborhood then the flooding position is updated. The neighbors and their positions are identified by the exchange of "Hello" protocol. The concept of route locking ensures that a node can return its packet to the last node when it cannot keep on sending the packet to the next node [6]. Once packets arrive at the nodes destination, then the end host broadcast a network information congregation packet to its hosts. The sending node computes the most excellent route in accordance with composed information as well as after that instantly starts to send data packets [7].

GRP provides a high-quality structure which can work at the same time with the strength of reactive routing protocol and proactive routing protocol which gathers network information at a sending node using a little organize overheads. The promising routes can be equipped by source nodes on the basis of the gathered information and it continuously transmits data packets even if the current route may be disconnected, it results in achieving fast packet transfer delay without unduly compromising on control overhead performance [8].

\section{RELATED WORK}

In 2012, Kaur and Singh [7] performed experiments for evaluation of proactive, reactive as well as hybrid routing protocols (OLSR, TORA and GRP) by Opnet simulator. According their results OLSR has highest throughput in each scenario in any case of high routing overhead and delay. TORA carry out the most unpleasant case at the same time as it reduce the control overhead with localizing the hosts. It was over and done with that performance of OLSR was most excellent in network load as well as throughput, while GRP excellent in term of delay as well as routing overhead. At the end performance of OLSR was well again than other protocols.

In 2012, Keshtgary and Babaiyan [9] studied the behaviour of AODV, OLSR, DSR and GRP MANET protocols. Their evaluations have been complete among OLSR, AODV, GRP and DSR protocols. According to their study they considered these constraint for the assessment e.g. network load, throughput, load, media access delay and delay. Their result showed DSR was the worst option as they consider four aforesaid metrics. In other metrics, OLSR perform better than DSR and AODV protocols while DSR was worst case routing protocol.

In 2012, Pragati and Nath [10] evaluated that the end-to-end delay and average metric has revealed that AODV, TORA average delay was better in case of end to end delay, however in LEACH, it was not as much of as contrast to TORA, AODV. The packet loss was show that many of packet losses in TORA, AODV was additional owing to overhead.

In 2012, Santhamurthy [11] held a logical survey on show of AODV, OLSR and TORA. The ending of the research was that OLSR was the best in high density network, AODV was best in packet delivery.

In 2012, Palta and Goyal [5] concluded the results to facilitate the OLSR are better in that set-up anywhere bandwidth is large. OLSR forever modernize their nodes consequently need much bandwidth used as compared with TORA on similar environment. Later than comparing the network performance of these protocols it was observed that OLSR performs better against TORA by increasing bandwidth. OLSR was yet again showing good results in delay and throughput than TORA by changing network mobile node.

In 2012, Gupta and Kaushik [12] analyzed the behaviour of different routine matrices for MANETs by different protocols. Simulations were done to compare these routing protocols. 
In 2012, Anjali and Singh [8] worked on the performance of AODV, GRP OLSR protocol correspondingly and evaluated the metrics e.g. Media Access Delay, Network Load, Throughput and Retransmission Attempts by changing number of nodes. They concluded that the GRP is showing better results than AODV and OLSR.

\section{PERFORMANCE MATRICES}

In MANET, a number of verities of physical matrices may be analyzing the performance of MANET protocol. The main performance metrics which are evaluated the performance of reactive, proactive and hybrid routing protocols are Throughput, Network Load, Retransmission Attempt and Media Access Delay by varying physical metrics that are No. of nodes, nodes speed and pause time.

\subsection{Simulation parameters used in OLSR, TORA and GRP routing protocols scenarios.}

Network area set in this research $1000 * 1000 \mathrm{~m}$, moreover network simulation time was carried out having $3600 \mathrm{sec}$. The main performance parameters are Throughput, Network Load, Retransmission Attempts as well as Media Access Delay by varying physical network metrics like No. of Nodes, Pause Time as well as Node Speed. The no. of nodes is taken 20, 40 and 60 . The pause time is 100,200 and $300 \mathrm{sec}$. The node speed is $10,20,30 \mathrm{~m} / \mathrm{s}$. While the Random way point of the mobility model is fixed by FTP traffic on high traffic load by MAC protocol IEEE 802.11.

In 2012, Kaur and Singh [7] focused only on Throughput, Network Load, Retransmission Attempts, Media Access Delay and nodes speed is fix that is 10 . They did not discuss the physical parameters e.g. nodes speed, Pause time and changing traffic type etc.

Table 1. Simulation Parameters

\begin{tabular}{|ll|}
\hline Parameter Name & Values \\
\hline Protocols & OLSR, TORA, GRP \\
\hline Simulator Model & OPNET 14.0 \\
\hline MAC Protocol & IEEE 802.11 \\
\hline Traffic Type & FTP \\
\hline Node Speed & $10,20,30 \mathrm{~m} / \mathrm{s}$ \\
\hline Pause Time & $100,200,300$ second \\
\hline No. of Nodes & $20,40,60$ \\
\hline Topology & Random Way point \\
\hline Time & 3600 second \\
\hline Mobility Speed & $10 \mathrm{~m} / \mathrm{s}$ Constant \\
\hline Area Size $\mathbf{x}$ & 1000 meters \\
\hline Area Size $\mathbf{y}$ & 1000 meters \\
\hline Node Movement & Random \\
\hline
\end{tabular}

\subsection{Application Configuration}

In OPNET 14.0 MODELER, the main application configuration is set of predefine rules which have many predefined libraries to built the network environment according to the requirement. Simulation for all fresh projects in aforesaid software, it is necessary to configure the application configuration. Mostly FTP is configured as high load.

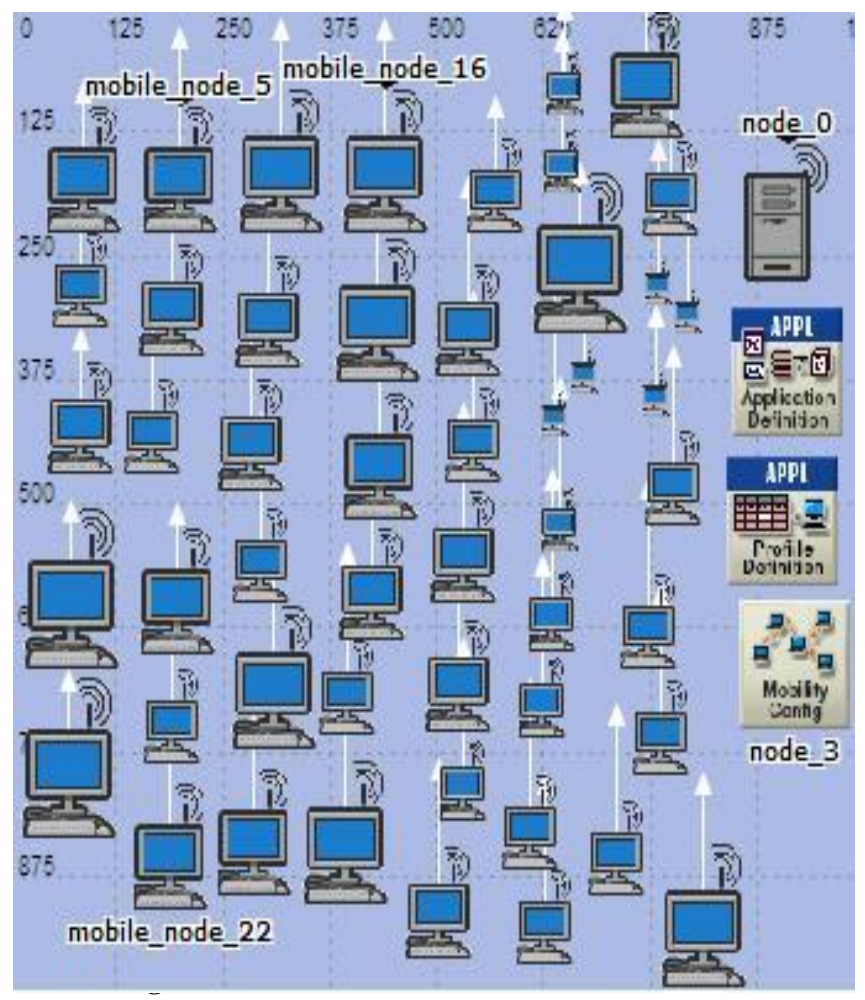

\subsection{OLSR, TORA and GRP throughput regarding nodes speed, pause time and network density}

The following scenarios are generating to assess the performance of throughput of OLSR, TORA and GRP routing protocols. In the first figure throughput of OLSR, TORA and GRP is evaluated regarding changing no. of nodes e.g. 20, 40 and 60 . In second figure throughput is analyzed by changing pause time e.g. 100, 200, 300 as well as in third figure illustrate the throughput changing nodes speed e.g 10, 20 and 30.

As shown in following figures 4 to 6 , OLSR perform very well and has maximum throughput regarding physical metrics e.g. No. of nodes, nodes speed and pause time. While TORA and GRP performance is low and same in all physical metrics scenarios. OLSR perform better than TORA and GRP. 


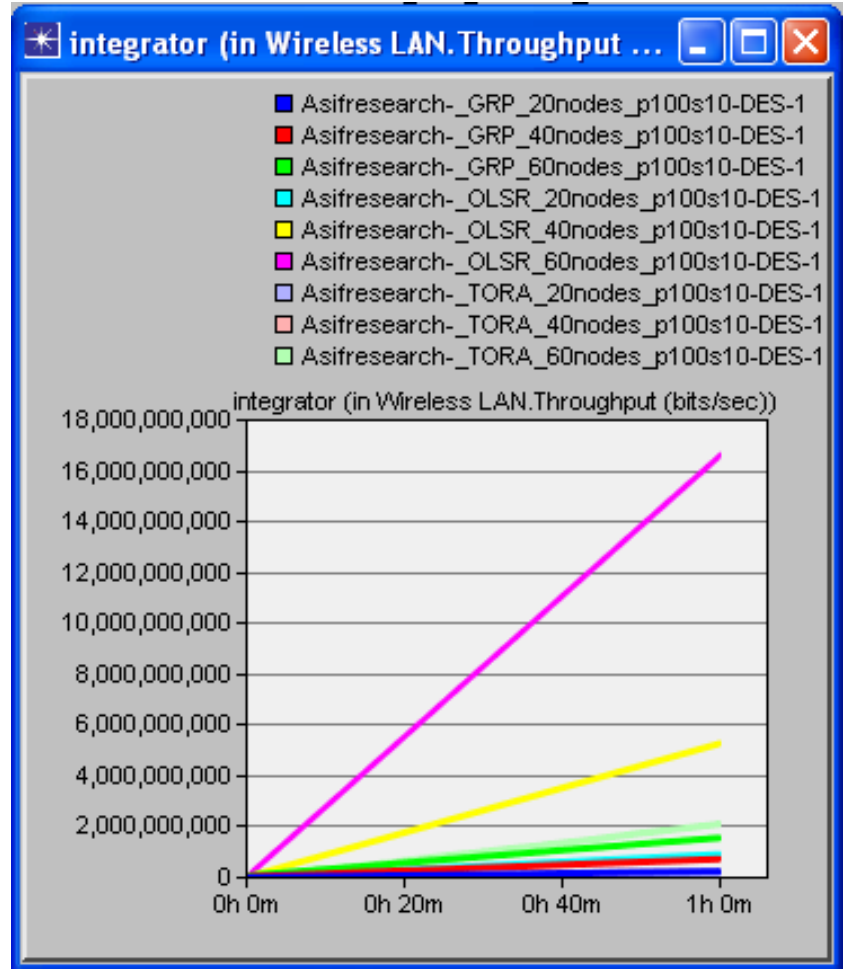

Fig 4: OLSR, TORA and GRP Throughput regarding no. of nodes

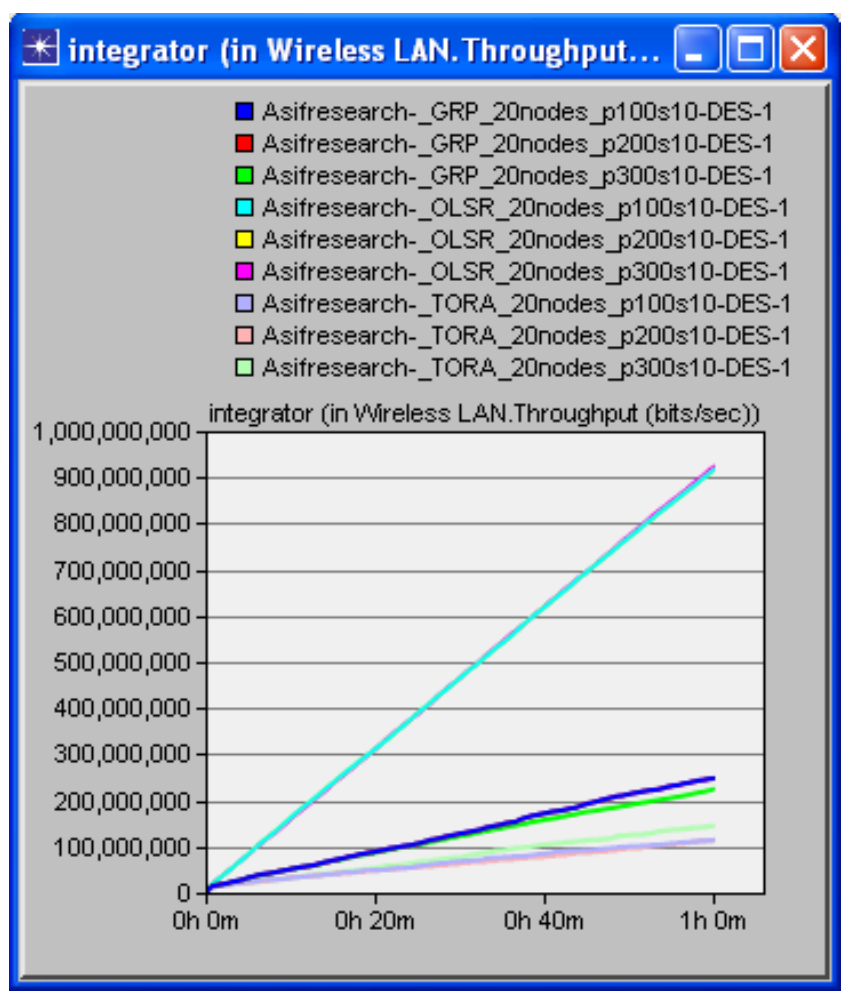

Fig 5: OLSR, TORA and GRP Throughput regarding pause time.

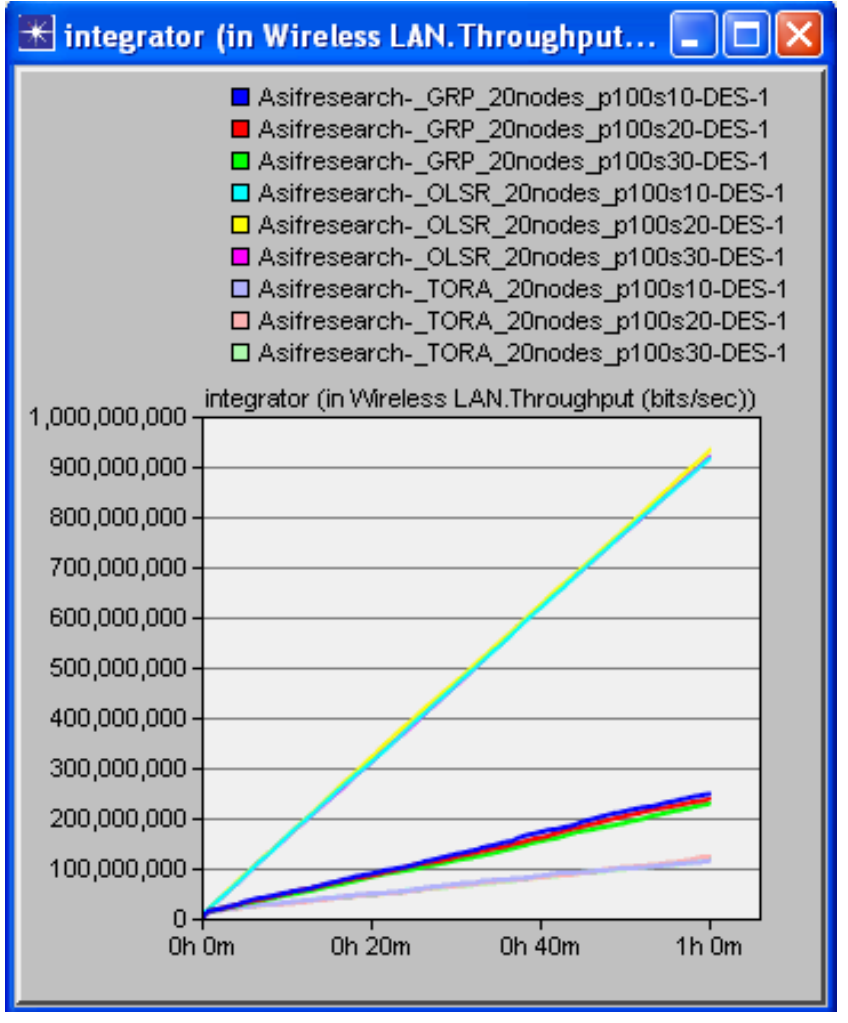

Fig 6: OLSR, TORA and GRP Throughput regarding nodes speed

\subsection{OLSR, TORA and GRP network load regarding nodes speed, pause time and network density}

The following three scenarios are generated to evaluate the network load (bits/sec) of OLSR, TORA and GRP routing protocols. In the first scenario network load (bits/sec) of OLSR, TORA and GRP is compared against varying nodes speed e.g 10, 20, 30. In second scenario network load (bits/sec) is observed by varying numbers of nodes e.g. 20, 40, 60 and third scenario shows the network load (bits/sec) varying pause time e.g. 100, 200, 300.

As illustrated in the following Figures 7 to 9 , it is observed that the maximum load of OLSR and TORA is approximately same. While the GRP performance has gone down as the No. of nodes speed increases. TORA performs well in 60 nodes network while performance of GRP is low as shown in Fig.8. The performance of TORA in pause time is outstanding as compared to OLSR and GRP. 


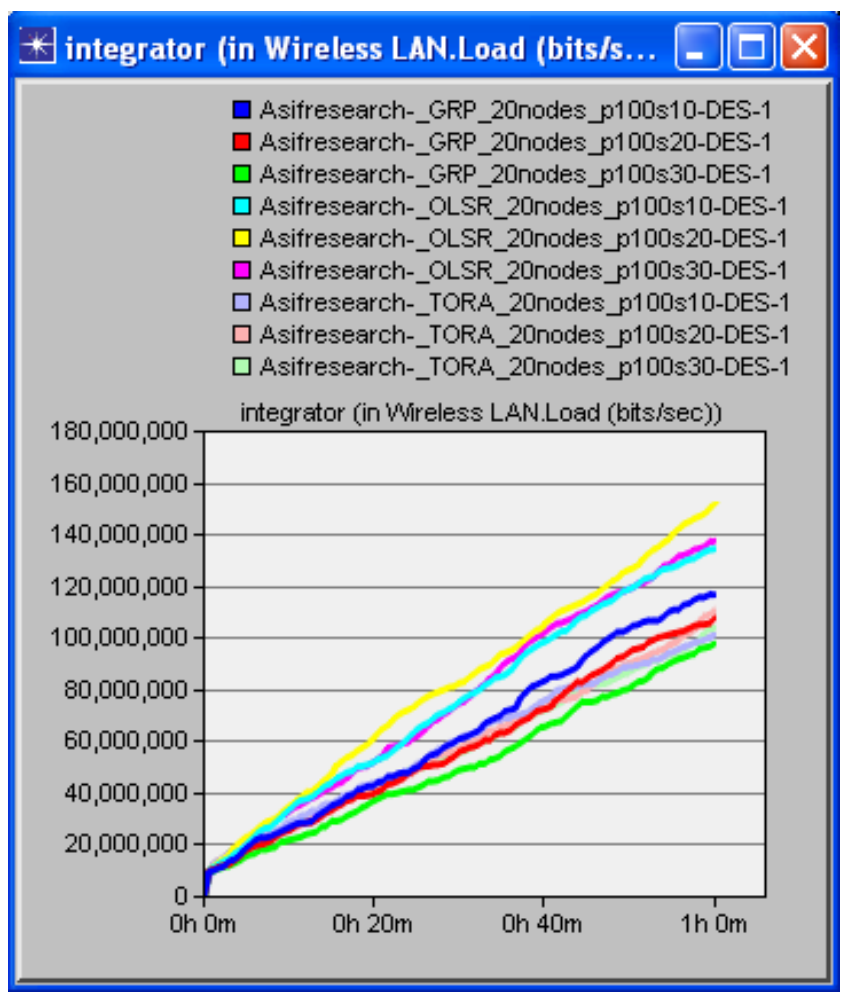

Fig 7: OLSR, TORA and GRP Network Load regarding nodes speed

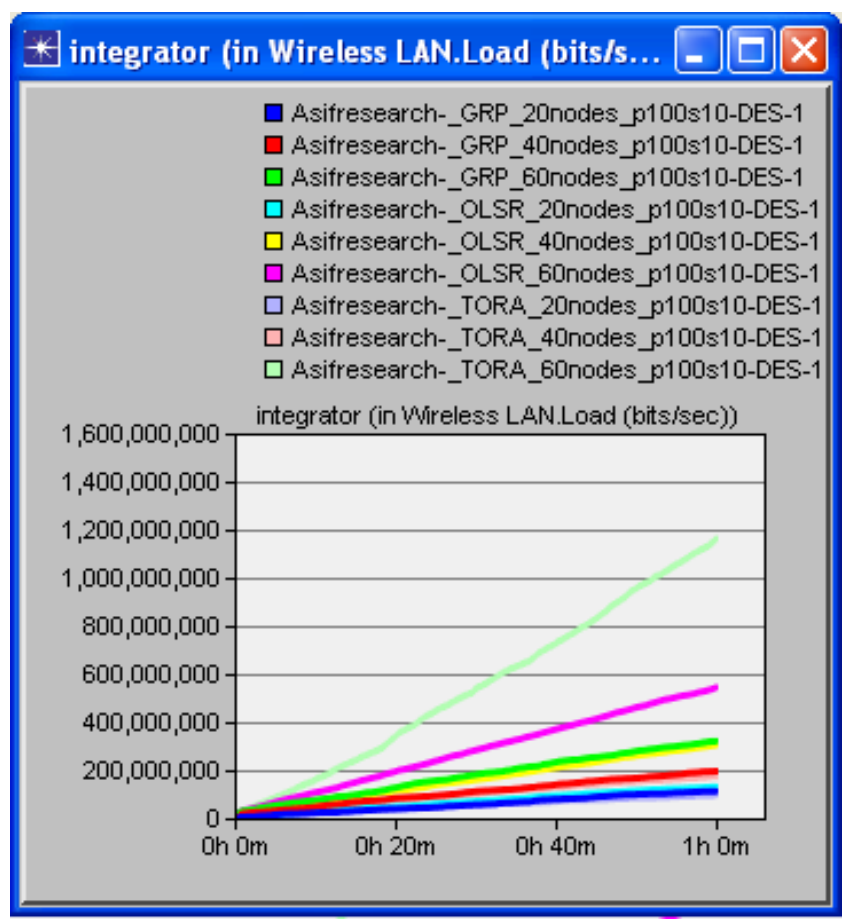

Fig 8: OLSR, TORA and GRP Network Load regarding no. of nodes

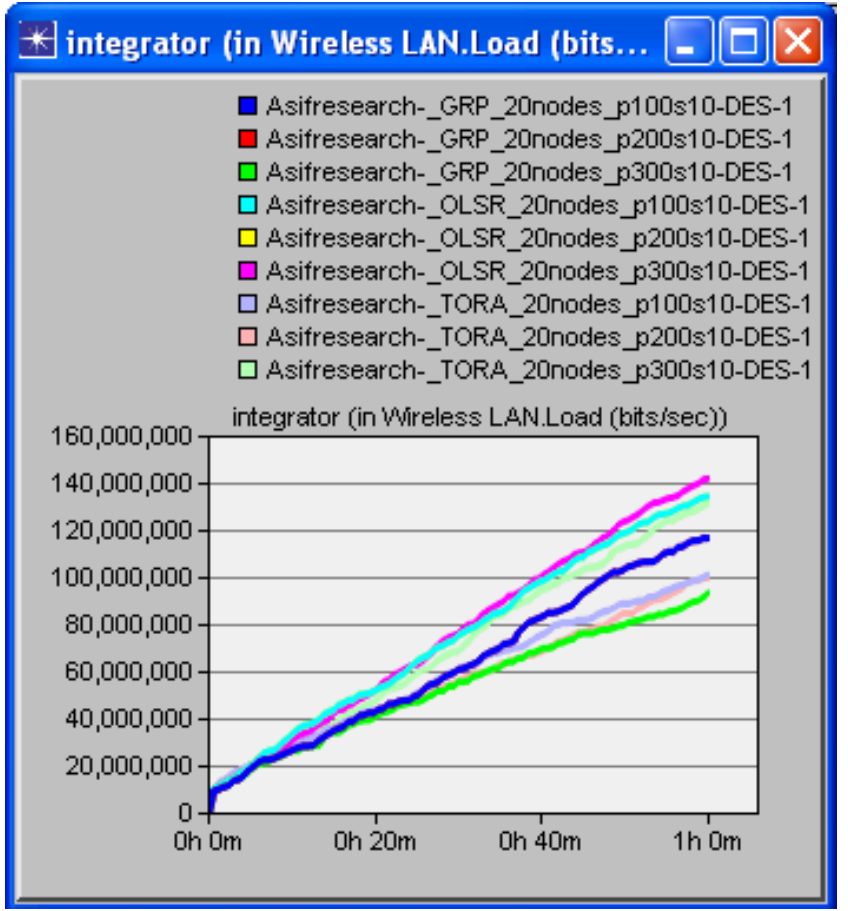

Fig 9: OLSR, TORA and GRP Network Load regarding pause time

\subsection{OLSR, TORA and GRP retransmission attempt regarding nodes speed, pause time and network density}

The following graphs represent the whole amount of bits/sec sent from wireless LAN layers to higher layers in every WLAN hosts of the network. Following scenarios are to estimate the retransmission attempt (packets) of OLSR, TORA and GRP routing protocols. In $1^{\text {st }}$ scenario retransmission attempt (packets) of OLSR, TORA and GRP is compared by changing pause time 100, 200 and 300. In second scenario retransmission attempt (packets) is observed by varying node speed 10,20 and 30. Third scenario shows the retransmission attempt (packets) by varying numbers of mobile nodes 20, 40 and 60 .

As illustrated in the following Figures 10 to 12, it is observed that the retransmission attempt (packets) of TORA is maximum. While OLSR and GRP maximum retransmission attempt (packets) is approximately same. In retransmission attempt (packets) TORA performs well in all the physical metrics. The performance of TORA in pause time and nodes speed is outstanding as compared to OLSR and GRP. 


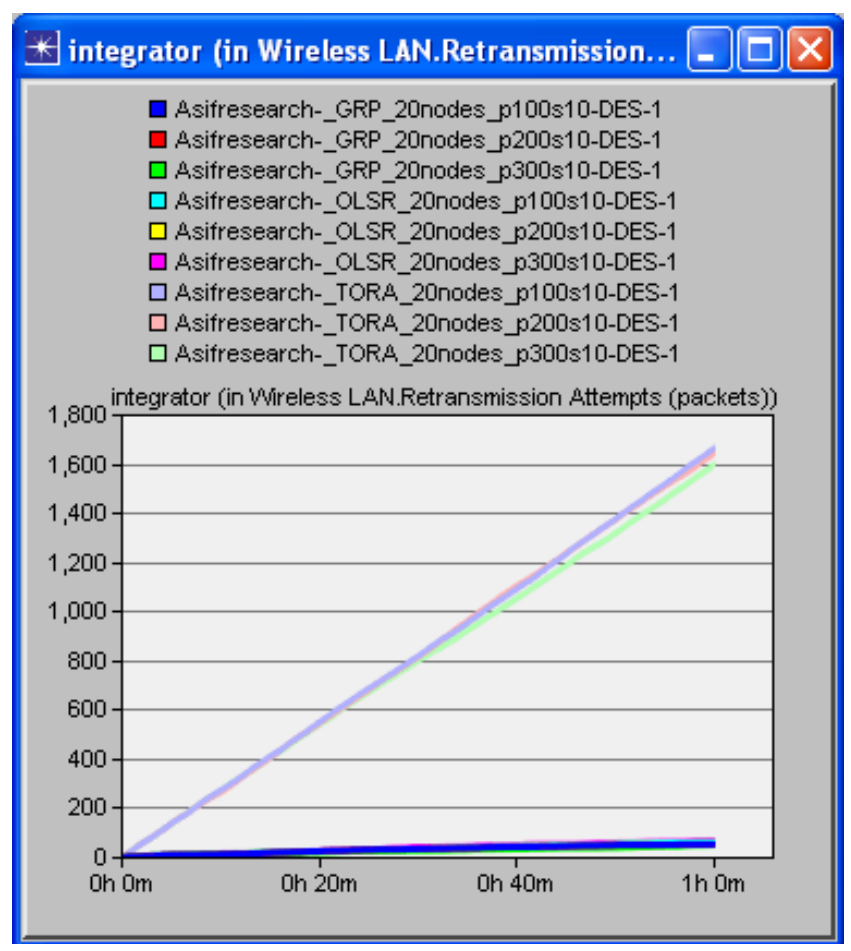

Fig 10: OLSR, TORA and GRP Retransmission attempt regarding pause time

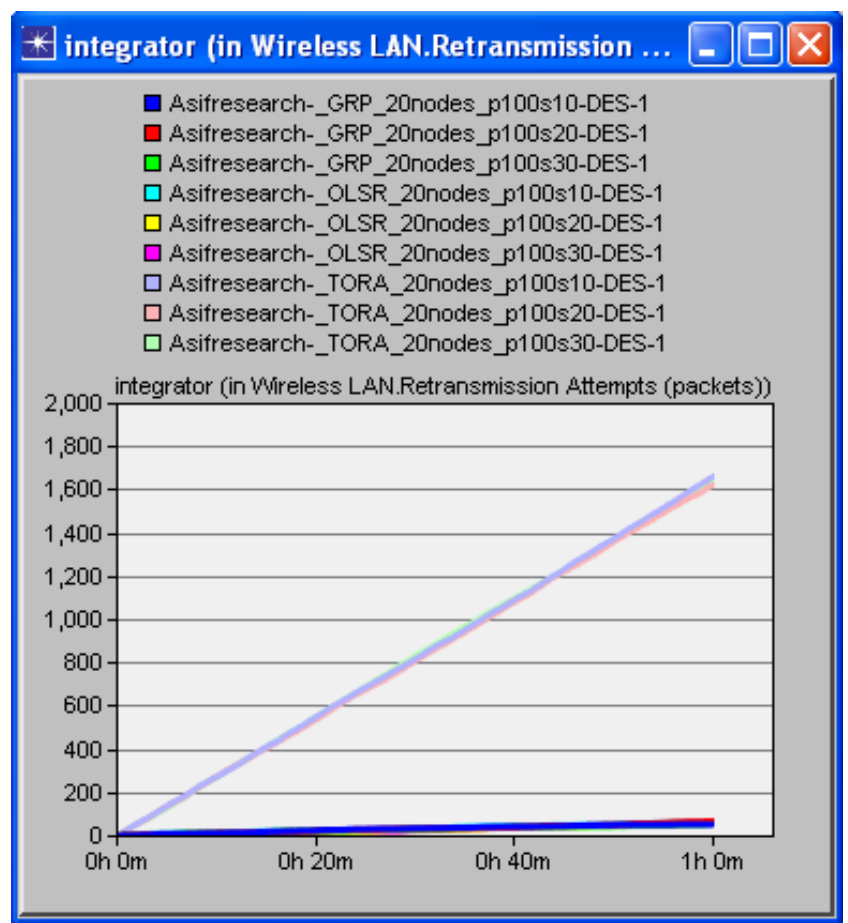

Fig 11: OLSR, TORA and GRP Retransmission attempt regarding nodes speed

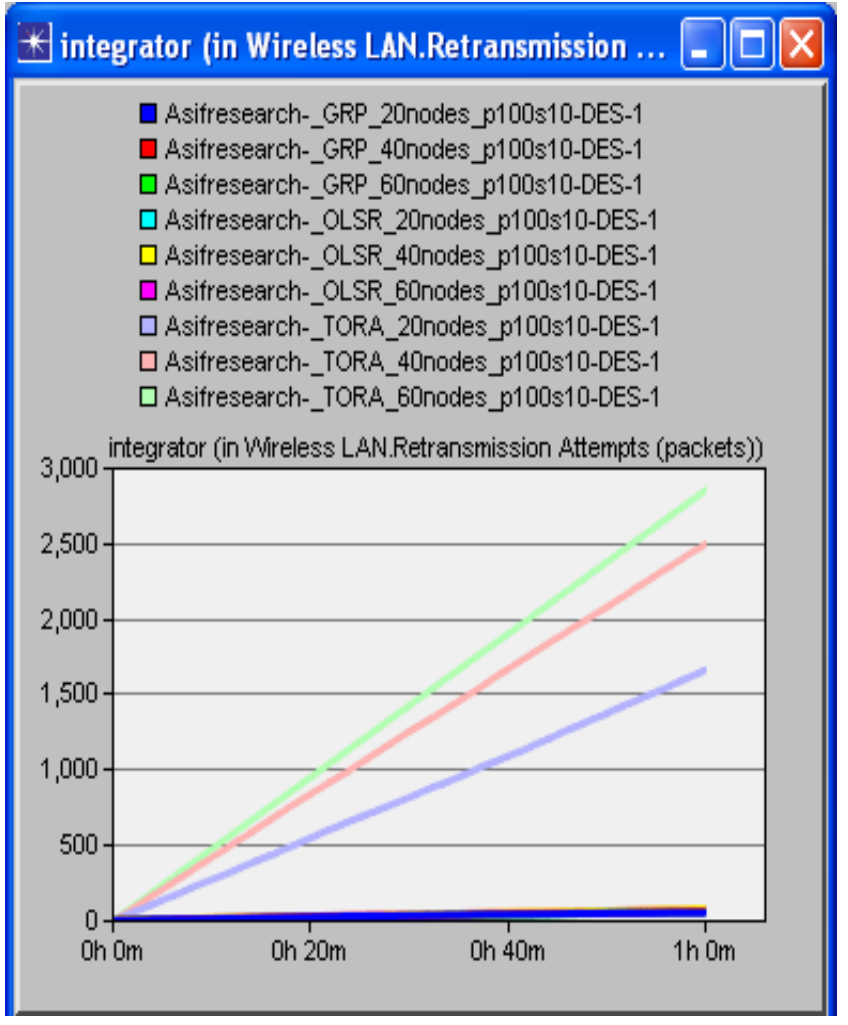

Fig 12: OLSR, TORA and GRP Retransmission attempt regarding no. of nodes

\subsection{OLSR, TORA and GRP media access delay regarding nodes speed, pause time and network density}

The following graphs represent the evaluation of the media access delay (Sec) of OLSR, TORA and GRP routing protocols. In the first scenario media access delay $(\mathrm{Sec})$ of aforesaid protocols is compared against varying node speed $(10,20,30 \mathrm{~m} / \mathrm{s})$. In second scenario media access delay (Sec) is observed by varying numbers of nodes e.g. 20, 40 and 60 and third scenario shows the media access delay (Sec) by varying pause time e.g. 100, 200 and $300 \mathrm{sec}$.

The following Figures 13 to 15 show the media access delay regarding Nodes speed, No. of nodes and pause time correspondingly. TORA send maximum packets from source to the destination. OLSR media access delay time is increased as the of no. of nodes increase. GRP has the smallest amount of delay when the number of nodes is increased. 


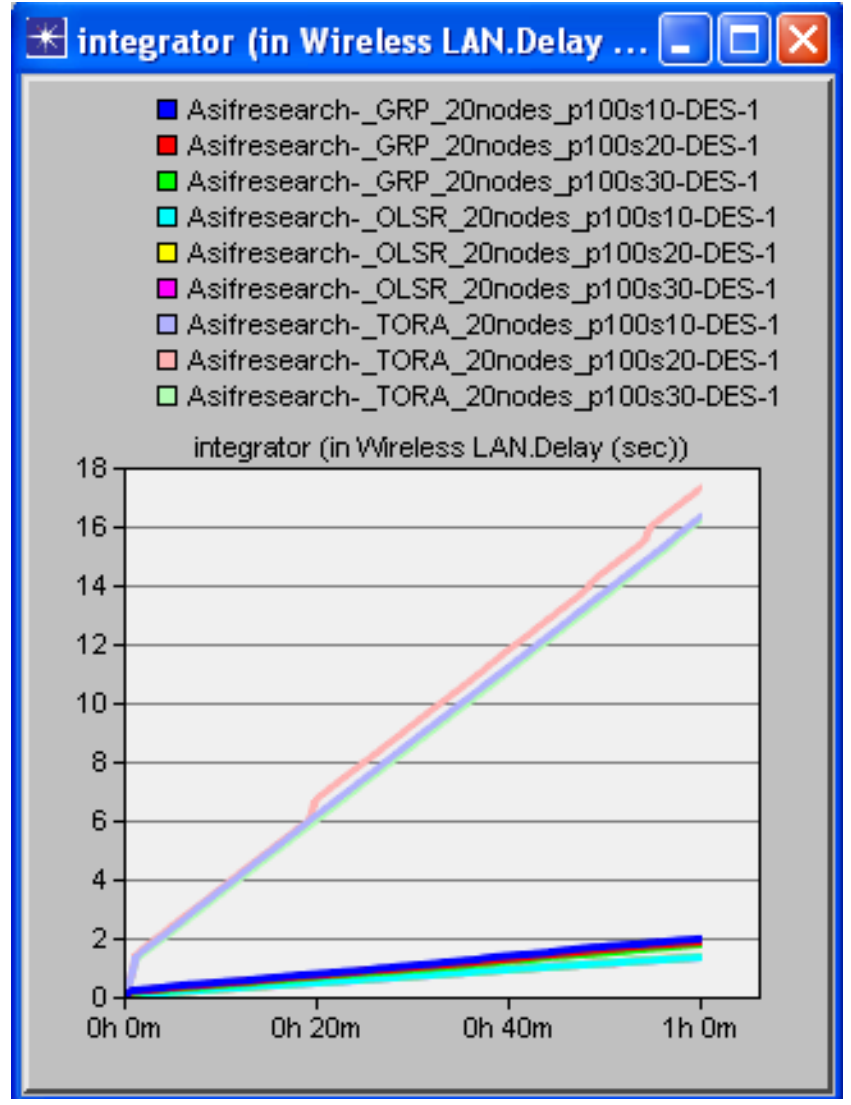

Fig 13: OLSR, TORA and GRP Media access delay regarding nodes speed

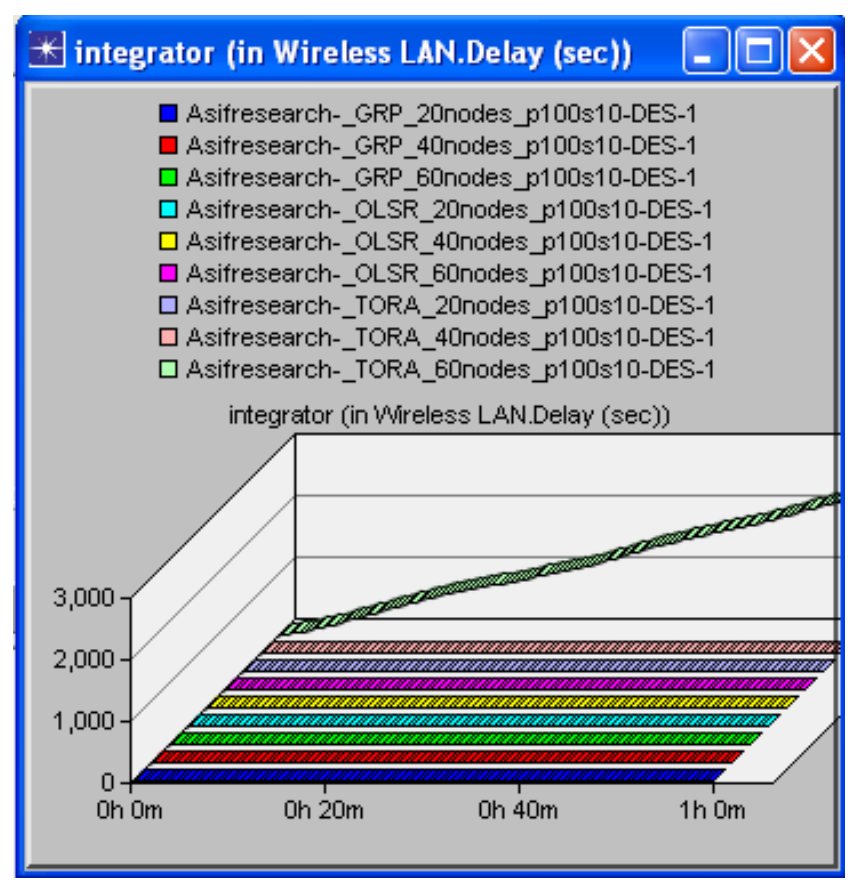

Fig 14: OLSR, TORA and GRP Media access delay regarding no. of nodes

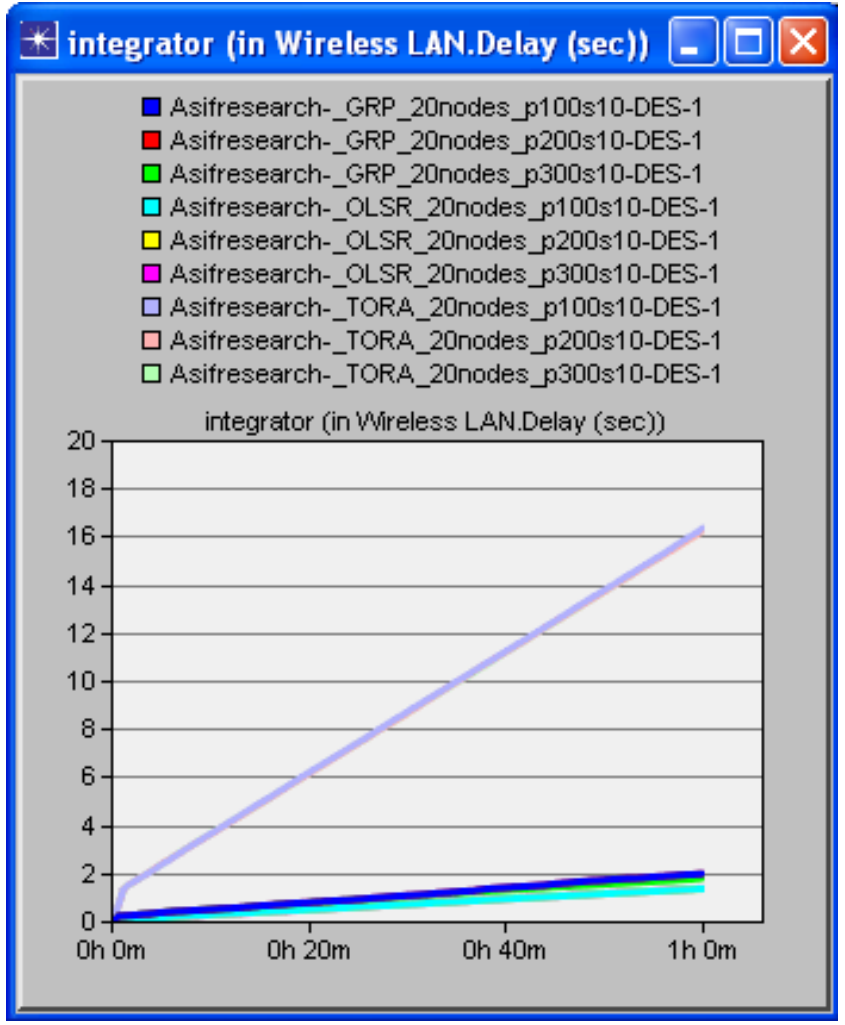

Fig 15: OLSR, TORA and GRP Media access delay regarding Pause Time

\section{CONCLUSION}

In this research, comparison of three ad hoc routing protocols (OLSR, TORA and GRP) is carried out in different circumstances by OPNET 14.0. The major performance metrics are throughput, network load, retransmission attempt and media access delay. These metrics are tested by varying the number of nodes, network speed and pause time applying FTP traffic. The simulation result shows that in throughput, OLSR performed outstanding regarding network density, pause time and network speeds in throughput. However TORA performance in throughput regarding network density and pause time is found better. In network load scenarios OLSR perform better as compare to TORA and GRP. OLSR and GRP performance in retransmission attempt regarding nodes speed is not better than TORA. In Media access delay scenario the performance of TORA is also better than OLSR and GRP. The simulations result show in this research that OLSR performs better than TORA and GRP regarding network density and pause time.

\section{FUTURE DIRECTIONS}

For every feasible parameter, this research work can be extended about the working of OLSR, TORA and GRP. This can also be extended if someone desire to restructure a network and he needs the guidelines for selection of OLSR, TORA and GRP regarding network performance comparison. In the future, wide complex simulations could be accepted using other performance metrics, in order to gain a more indepth performance analysis of the ad hoc routing protocols. Other new protocols performance could be studied too. 


\section{ACKNOWLEDGEMENT}

I sincerely thank my supervisor and my dearest friends for their support to write this paper. Without their right guidance this would not have been possible.

\section{REFERENCES}

8. Taneja S., A. Khush and A. Makkar, 2010. Experimental Analysis of DSR, AODV using Speed and Pause time. International Journal of Innovation, Management and Technology, 1 (5): 453-458.

9. Jaafar M. A., and Z. A. Zukarnain, 2009. Performance Comparisons of AODV, Secure AODV and Adaptive Secure AODV Routing Protocols in Free Attack Simulation Environment. European Journal of Scientific Research, 32 (3): 430-443.

10. Taneja S and A. Kush, 2010. A Survey of Routing Protocols in Mobile Ad Hoc Networks. International Journal of Innovation, Management and Technology, 1 (3): 279-285.

11. Lakhtaria K. I. and P. Patel, 2010. "Analyzing Zone Routing Protocol in MANET Applying Authentic Parameter". Global Journal of Computer Science and Technology. 10 (4): 114-118.

12. Palta, P. and S. Goyal, 2012. Comparison of OLSR and TORA Routing Protocols Using OPNET Modeler. International Journal of Engineering Research \& Technology (IJERT), 1(5): 1-6.

13. Aujla, G. S., \& Kang, S. S., 2013. Comprehensive Evaluation of AODV, DSR, GRP, OLSR and TORA Routing Protocols with varying number of nodes and traffic applications over MANETs, IOSR
Journal of Computer Engineering (IOSR-JCE), 9 (3): $54-61$

14. Kaur, H. and J. Singh, 2012. Performance comparison of OLSR, GRP and TORA using OPNET. International Journal of Advanced Research in Computer Science and Software Engineering, 2(10): 260-267.

15. Anjali and M. Singh, 2012. Performance Analysis of Proactive, Reactive and Hybrid MANET Routing Protocols on IEEE 802 . 11 Standard. International Journal of Computer Applications, 54(12): $1-8$.

16. Keshtgary, M. and V. Babaiyan, 2012. Performance Evaluation of Reactive , Proactive and Hybrid Routing Protocols in MANET. International Journal on Computer Science and Engineering (IJCSE), 4(02): 248-254.

17. Pragati Er. and R. Nath, 2012. Performance Evaluation of AODV, LEACH \& TORA Protocols through Simulation. International Journal of Advanced Research in Computer Science and Software Engineering, 2(7): 84-89.

18. Santhamurthy, T., 2012. A Quantitative Study and Comparison of AODV, OLSR and TORA Routing Protocols in MANET. IJCSI International Journal of Computer Science, 9(1): 364-369.

19. Gupta, M., and S. Kaushik, 2012. Performance Comparison Study of AODV, OLSR and TORA Routing Protocols for MANETS. International Journal Of Computational Engineering Research, 2(3): 704-711. 